\title{
Combined Measurement System for the Evaluation of Multi Causal Strain
}

\author{
Holger Steiner ${ }^{1}$, Dietmar Reinert ${ }^{1}$, and Norbert Jung ${ }^{2}$ \\ ${ }^{1}$ BGIA - Institute for Occupational Health and Safety of the German Social Accident Insur- \\ ance, Alte Heerstraße 111, 53757 Sankt Augustin, Germany \\ ${ }^{2}$ University of Applied Sciences Bonn-Rhein-Sieg, \\ Grantham-Allee 20, 53757 Sankt Augustin, Germany \\ \{mail@holgersteiner.de, dietmar.reinertadguv.de, \\ norbert.jung@h-brs.de \}
}

\begin{abstract}
This work addresses the problem of measuring psychological strain in humans by the use of physiological data. The aim of the work is the research, development and evaluation of a measurement system for the acquisition of such data from humans and the differentiation of psychological and physical strain with the help of machine learning algorithms. The developed system records and analyzes the ECG, the EMG, as well as the skin conductance, and combines these physiological parameters with the subject's physical activity. The main purpose of this measurement system is to assess both types of strain in employees at their workplaces.
\end{abstract}

Keywords: multi causal strain, stress, strain, ambulatory monitoring, physiological monitoring, physical activity, decision tree learning, machine learning.

\section{Introduction}

Strain can have multiple causes and consequences, which might be a risk for the health of employees and result in high costs for health insurance companies (see [9]). Researching the strain employees encounter during their work can help to prevent or reduce the strain, for example by modifying the working environment or tools, leading to safer working conditions for the employees and reduced costs for the insurance companies.

In this context, different types of strain have to be distinguished, as strain can be physical (that is, the body of the subjects is affected directly) or psychological: high mental workload or an emotionally stressful working environment might lead to psychological problems, such as depression, but can also be the cause for physical problems, such as illness or back pain.

To distinguish between the different types of strain and to classify their magnitude and their influence on employees, a complex measurement system is necessary, as different parameters for physical and psychological or emotional strain have to be acquired simultaneously. The research and development of such a measurement system is the aim of this work. 


\subsection{Measurement of Physical Strain}

For the measurement of physical strain and physical activity, a well-suited measurement system is available that has been developed at the BGIA Institute for Occupational Health and Safety: the CUELA system ([2]). CUELA is a German abbreviation and stands for "Computer-unterstützte Erfassung und Langzeit-Analyse von Belastungen des Muskel-Skelett-Systems", which can be translated as "computer-assisted measurement and long-term analysis of strain on the musculoskeletal system". The system has been used in a large number of applications since the year 1997, primarily for the assessment of physical strain that employees encounter at their workplaces. The standard CUELA system is a sensor suite that can be worn over the working clothes. It is able to measure physical strain of a subject on the basis of the body posture and movements. It uses mechanical sensors to measure the angles of the thoracic and lumbar spine, the torsion of the back, as well as the angles of the hip and knee joints.

On the basis of the standard CUELA system, a modified measurement system called "CUELA Activity" has been developed in the last two years by Weber (see [12]). It uses accelerometers and gyroscopes to measure the actual body posture and movement instead of the mechanical sensors of the standard CUELA system, thus reducing the weight, size and complexity of the sensor suite significantly. The main purpose of the Activity system is to evaluate the actual activity of a subject in terms of both the type of the performed activity and the speed or intensity of movement.

The data that is measured by the system is recorded by a so-called "datalogger" and analyzed at a later time with a software called WIDAAN (which stands for "Winkel-Daten-Analyse", or "angle data analysis", respectively). WIDAAN runs on Windows computers and allows to display the recorded data in the form of graphs over time, as well as in the form of an animated 3D-skeleton figure. WIDAAN is also able to synchronize the measured data with a video recording. It handles every sensor or calculation result as a "channel", which can be viewed as a chart or graph over the time, and allows to synchronize, mark and edit single or multiple data channels at once ([2], [3]). The design of the WIDAAN software allows to extend it with new data channels or new "plug-in" components for the calculation of strain data easily.

\subsection{Measurement of Psychological Strain}

It is obvious that psychological strain can not be measured by using physical data alone. Therefore, the main idea of this work is to develop an additional physiological measurement system that can be combined with either one of the already existing CUELA systems. Appropriate physiological parameters and sensors that are suited to acquire these parameters have been researched in the scope of this work.

Compared to the physical activity, physiological parameters represent a more direct way to measure and evaluate the strain of a subject. In general, these parameters measure the reaction of the human body to both physical and psychological strain of any kind. The results that can be achieved by the assessment of these parameters are always subjective to a certain extend, as the subject's personal fitness and individual perception of the stressor have an influence on the physiological parameters (see [6]). The heart rate and the heart rate variability, which are both measured by an 
electrocardiogram (ECG), the electrodermal activity (skin conductance response, respectively) and the muscle activity in the Trapezius muscle, which is measured by an electromyogram (EMG), have been selected due to their importance for the measurement of strain (compare to [4], [10], [11], [14], [15]).

While the heart rate is strongly correlated with the overall strain of the body, the heart rate variability, which represents the variations of the heart rate from beat to beat, can be used to assess mental strain ([6]). It is typically assessed in terms of the "root mean square of successive differences" (RMSSD) and the standard deviation of the beat-to-beat-intervals (SDRR), which are both measured in milliseconds. In contrast to this, the skin conductance response allows the detection of emotional strain and arousal (see [15]). The skin conductance (that is, the ability of the skin to conduct electricity) is measured in Siemens and depends on the amount of sweat in the sweat glands of the skin. The activity of the Trapezius muscle, which is situated in the shoulder and neck region of the human body, is also known to be an indicator for strain, especially when activity is detected during phases without body movements (compare to [10], [11]).

To provide context data in addition to the physiological parameters, the physical activity, which is measured by the use of either the standard CUELA system or the Activity system, is recorded simultaneously.

\section{Methods}

\subsection{Basic Idea}

As previously described, there are several parameters that are of interest for the measurement of both psychological and physical strain. The physical activity can be acquired and analyzed in detail by the use of the CUELA system in its different variants. However, in the scope of this work, only the CUELA Activity system has been used, as it already provides a parameter called "Physical Activity Intensity (PAI)", which is well-suited for this purpose. Therefore, this system is a very good basis for the development of the proposed multi causal strain measurement system. The implementation of microcontroller-based intelligent sensor systems for the acquisition and analysis of the physiological parameters, which can not be acquired by the CUELA system so far, and the integration of these sensor systems into the CUELA system is the basic idea of this work.

\subsection{System Concept}

The concept for the combined measurement system is shown in figure 1. The first and most important part of the system concept is represented by the physiological sensor systems themselves, which are shown in the upper left corner of the figure. On the hardware side, they consist of the actual sensor for the acquisition of the physiological signal, electrical circuitry and components for the analog signal conditioning, as well as one microcontroller IC-chip. On the software side, several functions are implemented on the microcontroller to deal with all signal processing steps that are applied on the digitalized signal. The developed microcontroller-based sensor systems are able to filter, digitalize and analyze the acquired sensor readings autonomously. 


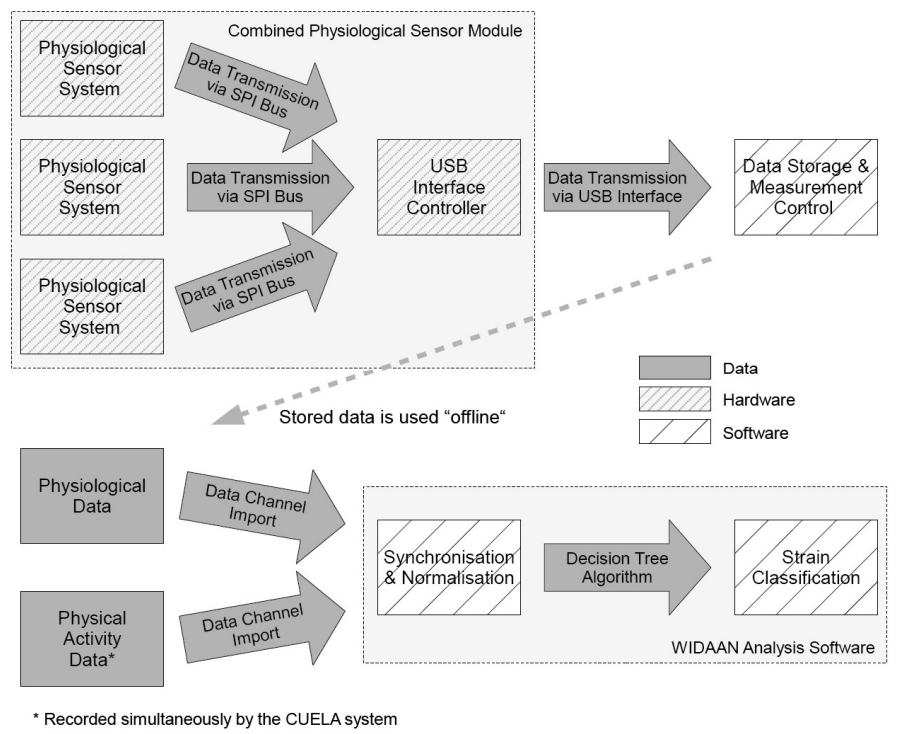

Fig. 1. System concept and data flow chart. Data and its flow is shown in dark grey, while hardware components are shown in (dashed) light grey and software components in (dashed) white.

The physiological sensor systems are combined on one hardware module (represented by the area marked in light grey), together with an USB interface controller. The sensor systems transmit their data to the USB controller by using a SPI bus interface. The USB controller unites the data and transmits it over its USB bus interface to a host device, which stores the data and controls the measurement process by sending control commands back to the physiological sensor systems (via the USB controller). This host device, which is called "datalogger" in conjunction with the CUELA system, can be any personal computer or notebook, as well as a personal digital assistant (PDA) or handheld computer, or even a mobile phone with USB host capabilities and sufficient memory to store the entire measurement data. Therefore, only a software tool that is able to manage the storage of the acquired data and that allows a user to control the measurement system has to be developed in order to implement this component of the system.

From this point on, the data is stored and available for "offline" use, which means that it can be viewed and analyzed after the measurement has been completed. All further components of the system work with this stored data. They are implemented completely in software, which runs on typical computer hardware like a desktop PC or a notebook. The basis for the software implementation is the WIDAAN analysis software of the CUELA system, which provides a graphical user interface to view, edit and analyze the recorded data and to synchronize the different data streams. The stored data from both the physiological and the physical sensor systems is imported into WIDAAN and new data channels are created for each sensor.

Afterwards, a decision tree algorithm is used to classify the data in the course of time with respect to the type and amount of strain that the subject experienced. Again, 
a new data channel is created in WIDAAN and the results of the algorithm's calculations are stored and graphically represented in this channel. In order to develop appropriate decision rules for the classification of the strain data, a learning algorithm is used on suited learning data. The resulting strain classification method is the final step of the system concept and represents the result of the approach that is proposed in this work.

\subsection{Details of the Physiological Measurement System}

In the physiological sensor systems, the physiological signals that are acquired by the use of electrodes are "conditioned" before they are digitalized, which means that they are amplified and filtered in order to remove noise and to limit their bandwidth by attenuating frequencies above a certain threshold. This is necessary in order to digitalize the signals correctly: according to the Nyquist-Shannon sampling theorem, the sampling rate, which is applied during the digitalization, has to be more than twice as high as the highest frequency occurring in the signal ([8]). If this theorem is broken, disturbing aliasing effects are the consequence. Therefore, a sampling rate is chosen which is sufficiently high for the respective sensor system.

After the digitalization, an additional digital notch filter is applied on the signal to remove $50 \mathrm{~Hz}$ and $60 \mathrm{~Hz}$ noise caused by main power lines. This noise gets easily picked up by the human body and can not be attenuated sufficiently by the analog signal conditioning process, as it is too close to the useful signals' frequency ranges. In the ECG sensor, the resulting signal is analyzed by an algorithm that detects the socalled R-wave, which is the highest peak in the signal. The R-wave marks the exact moment of the heart beat and can therefore be used to calculate the heart rate, as well as the heart rate variability, by measuring the time interval between two successive Rwaves. In the skin conductance sensor, the readings are derived in order to gain the skin conductance response. In contrast to this, the readings of the EMG sensor are used without further calculations.

The hardware components of the sensor systems are implemented on one single circuit board, together with an USB controller, which gathers the data from all sensor systems and transmits it to a connected datalogger, as proposed in the system concept. The complete sensor module is powered by the datalogger via the USB connection and small enough to avoid hindering the subject in any way.

\subsection{Data Classification}

The data that is recorded by the combined measurement system has to be analyzed as a whole in order to evaluate the type and amount of strain that the subject experienced during the measurement. This can be done manually by viewing and evaluating the data in numbers or as a graph, but this approach would be much too time-consuming to be performed for long time measurements or for a series of measurements with different subjects. Therefore, an automatic and autonomously working algorithm is needed that is able to perform this task in less time.

On the basis of the combined measurement system, algorithms for the differentiation between physical and psychological strain have been researched. A machine learning algorithm, which is able to automatically classify new data according to rules 
that it has learned before, promises to be an optimal solution for the given task. In order to gain training data for the use in such learning algorithms, a number of different strain classes have been specified and for each of these classes, a respective working scenario has been created with the intention to induce the specific amounts of psychological and / or physical strain. These scenarios include parts of both low and high physical and mental workloads, which are induced by a bicycle ergometer and a computer-based math and color recognition test (the so-called Stroop color test) that is based on the dual-task-paradigm (see [13]). Table 1 presents the nine specified strain classes and a description of the respective working scenario.

Table 1. Strain classes and the respective working scenarios for the generation of training data

\begin{tabular}{|l|l|}
\hline Strain Class & Description \\
\hline 1 - no strain & $\begin{array}{l}\text { Resting phase, also used to determine baseline values } \\
\text { for reference. At least ten minutes. }\end{array}$ \\
\hline 2 - low psychological strain only & $\begin{array}{l}\text { Combination of simple mathematical and colour exer- } \\
\text { cises with a fair solving time of } 15 \text { seconds. }\end{array}$ \\
\hline 3 - high psychological strain only & $\begin{array}{l}\text { Combination of difficult mathematical and colour exer- } \\
\text { cises with a stronger limited solving time of } 10 \text { seconds. }\end{array}$ \\
\hline 5 - low physical strain only & Cycling with a low workload of approx. 50 Watts. \\
\hline 6 - low physical and high psychological strain & $\begin{array}{l}\text { Cycling with low workload and solving of difficult math- } \\
\text { ematical and colour exercises, } 10 \text { s solving time. }\end{array}$ \\
\hline 7 - high physical only & Cycling with higher workload of approx. 150 Watts. \\
\hline 8 - high physical and low psychological strain & $\begin{array}{l}\text { Cycling with high workload and solving of simple math- } \\
\text { ematical and colour exercises, } 15 \text { s solving time. }\end{array}$ \\
\hline
\end{tabular}

For the generation of data for the specified strain classes, a respective measurement setup has been developed and performed by several subjects. The resulting data has been analyzed and split up in a training and a test data set. A machine learning algorithm is applied on these data sets in order to find attributes and rules for the reliable classification of the data with respect to type and amount of strain. Due to the given characteristics of the input data (a fixed set of attribute-value pairs with numerical values, possibly containing errors or missing values due to incorrect sensor readings or loss of data), a decision tree learning algorithm was selected for this purpose (see [4], [7]). In decision tree learning, the data is split up by a number of simple decisions that together form a tree: for every data set, decision rules are applied following one path along the edges of the tree until a leave, which represents one of the specified classes, is reached. This type of learning algorithm produces static decision 
rules for the data classification, which can easily be implemented in the analysis software in order to perform the data classification automatically. The resulting decision rules will classify each of the data sets into exactly one of the previously defined (disjoint) categories.

\section{Results}

The proposed physiological measurement system has been evaluated in terms of the theoretical and technical implementation of the combined sensor system, as well as by a functional evaluation with respect to the practical use of the system. The results of the evaluation showed that the sensor modules are working correctly and produce good and sufficiently reliable results, even if the subject is in motion.

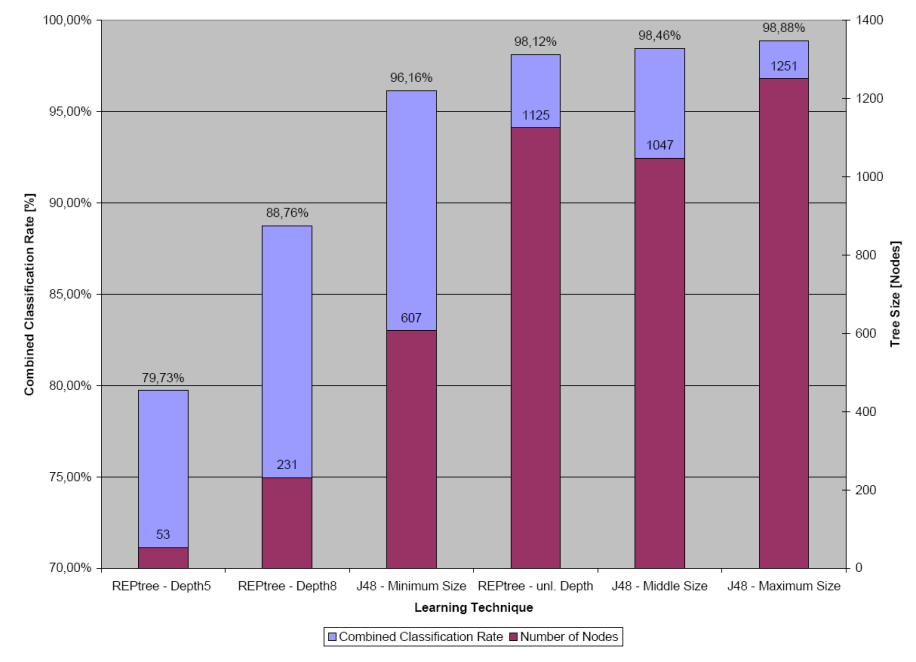

Fig. 2. The performance in terms of the combined classification rate (left Y-axis) of different decision tree learning techniques ( $X$-axis), which were applied on the joint data set of two subjects, compared to the respective tree size (right Y-axis)

Two data sets from subjects, who performed the specified measurement setup with the nine different working scenarios, were chosen to be used as training data for the learning algorithm. The decision tree learning algorithm delivers a very good performance for the classification of the data of each subject. Correct classification rates of more than $90 \%$ can be achieved even by comparably small decision trees with about 50 nodes (or decisions, respectively), which can easily be implemented in an analysis software. By using more complex trees with more than 500 nodes, correct classification rates of up to $98 \%$ could be achieved.

In order to find a classification that can be used for both subjects, the data sets were combined and the algorithm was applied on this combined data set. It was found that the correct classification rate is almost identical to the results of the algorithm for 
just one single data set. Unfortunately, this is achieved by a significant increase of the trees' sizes. However, if the tree's depth is limited to a fixed value (which is possible by modifying the algorithm's parameters), the size of the resulting trees is only moderately increased while still providing a good classification rate. Figure 2 shows the performance of different decision trees on the joint data set compared to their respective sizes.

\section{Discussion}

This work addressed the problem of measuring both psychological and physical strain in humans, especially in employees at their workplaces, and of differentiating between the types of strain the subject encountered. On the basis of intelligent sensor systems, a combined measurement system for the acquisition of physiological parameters has been designed and implemented. This measurement system can be used in conjunction with the CUELA system in order to acquire both physiological and physical parameters of a subject and all acquired data can easily be combined by the use of a single analysis software.

In order to automatically classify the combined data with respect to the type of strain that the subject encountered, the use of machine learning algorithms has been proposed. Nine different strain classes have been specified and respective strain induction scenarios have been performed by several subjects in order to gain training data with a known combination of strain for the use in the learning algorithm. It was shown that the decision tree learning algorithms deliver a good performance for the classification of the data. However, to achieve reliable classification results for unknown data that has been acquired from a new subject, using a tree that has been learned with the data from only one subject is not sufficient.

A decision tree that was learned with data from two subjects achieved a constantly high correct classification rate for both subjects, which is very promising with regard to a more general applicability of this method. This indicates that in order to find a decision tree, which can be applied successfully to the data of arbitrary subjects, training data from a large number of subjects has to be collected. This requires an additional study and further research in order to find convenient ways of normalizing the data, as well as the design of respective calibration phases that have to be performed by each new subject.

A different approach is to implement a complete decision tree learning algorithm into the data analysis software that learns a new decision tree for every new subject. This would require to extend the calibration phase that has to be performed by each subject, as it has to include all specified strain induction scenarios. Advantages of this approach are that the data does not have to be normalized and that it is not necessary to collect training data from a large number of subjects in advance. Additionally, the results of the classification algorithm might be better, as the distinctive features of each class are based on the specific subject's data only. However, the feasibility of both approaches has to be evaluated in detail in future work. 


\section{References}

1. Cutmore, T., James, D.A.: Sensors and Sensor Systems for Psychophysiological Monitoring: A Review of Current Trends. Journal of Psychophysiology 21(1), 51-71 (2007)

2. Ellegast, R.-P., Hermanns, I.: Einsatz des Messsystems CUELA zur Erfassung und Bewertung physischer Arbeitsbelastungen. BGIA - Institute for Occupational Health and Safety of the German Social Accident Insurance, Sankt Augustin, (2006) http: / / www.hvbg.de/d/bia/fac/ergonomie/pdf/cuela.pdf

3. Hermanns, I., Post, M.: Das CUELA-Messsystem. Information des Berufsgenossenschaftlichen Instituts für Arbeitsschutz - BGIA, Sankt Augustin (2003), http://www.hvbg.de/d/bia/fac/ergonomie/pdf/text1a.pdf

4. Mitchell, T.M.: Machine Learning. McGraw-Hill, Columbus (1997)

5. Myrtek, M., Fichtler, A., Strittmatter, M., Brügner, G.: Stress and Strain of Blue and White Collar Workers During Work and Leisure Time: Results of Psychophysiological and Behavioral Monitoring. Applied Ergonomics 30, 341-351 (1999)

6. Myrtek, M., Foerster, F.: On-Line Measurement of Additional Heart Rate - Methodology and Applications. In: Fahrenberg, J., Myrtek, M. (eds.) Progress in Ambulatory Assessment - Computer-Assisted Psychological and Psychophysiological Methods in Monitoring and Field Studies, pp. 399-414. Hogrefe \& Huber Publishers, Göttingen (2001)

7. Russel, S., Norvig, P.: Künstliche Intelligenz. Prentice Hall / Pearson Education, New Jersey (2004)

8. Smith, S.W.: The Scientist and Engineer's Guide to Digital Signal Processing. California Technical Publishing, San Diego (1999)

9. Steiner, H.: Erfassung von physiologischen Daten mit einem Embedded Controller. BGIA - Institute for Occupational Health and Safety of the German Social Accident Insurance / University of Applied Sciences Bonn-Rhein-Sieg, Sankt Augustin (2006)

http://www.inf.fh-bonn-rhein-sieg.de/data/ informatik/

fb_informatik/personen/reinert/steiner.pdf

10. Taelman, J., Adriaensen, T., van der Horst, C., Linz, T., Spaepen, A.: Textile Integrated Contactless EMG Sensing for Stress Analysis. In: Engineering in Medicine and Biology Society, EMBS 2007, 29th Annual International Conference of the IEEE, pp. 3966-3969. IEEE Press, New York (2007)

11. Vogt, J., Kastner, M.: Psychophysiological Monitoring of Air Traffic Controllers: Exploration, Simulation, Validation. In: Fahrenberg, J., Myrtek, M. (eds.) Progress in Ambulatory Assessment - Computer-Assisted Psychological and Psychophysiological Methods in Monitoring and Field Studies, pp. 455-476. Hogrefe \& Huber Publishers, Göttingen (2001)

12. Weber, B., Hermanns, I., Ellegast, R.P., Kleinert, J.: Assessment Of Physical Activity at Workplaces. In: Bust, P. (ed.) Contemporary Ergonomics. Taylor \& Francis, Oxfordshire (2008)

13. Wickens, C.D., Gordon, S.E., Liu, Y.: An Introduction to Human Factors Engineering. Addison Wesley Longman, Harlow (1998)

14. Wilson, G.: In-Flight Psychophysiological Monitoring. In: Fahrenberg, J., Myrtek, M. (eds.) Progress in Ambulatory Assessment - Computer-Assisted Psychological and Psychophysiological Methods in Monitoring and Field Studies, pp. 435-454. Hogrefe \& Huber Publishers, Göttingen (2001)

15. Wilhelm, F.H., Pfaltz, M.C., Grossman, P., Roth, W.T.: Distinguishing Emotional From Physical Activation. In: Ambulatory Psychophysiological Monitoring, Rocky Mountain Bioengineering Symposium \& International ISA Biomedical Sciences Instrumentation Symposium (2006) 